anales de psicología, 2016, vol. 32, $\mathrm{n}^{\circ} 2$ (mayo), 545-554 http://dx.doi.org/10.6018/analesps.32.2.205661
C) Copyright 2016: Servicio de Publicaciones de la Universidad de Murcia. Murcia (España) ISSN edición impresa: 0212-9728. ISSN edición web (http://revistas.um.es/analesps): 1695-2294

\title{
Desarrollo y validación de la Escala de Expectativas de Futuro en la Adolescencia (EEFA)
}

\author{
Yolanda Sánchez-Sandoval* y Laura Verdugo
}

Universidad de Cádiz (España).

\begin{abstract}
Resumen: El objetivo de este estudio es diseñar y validar el instrumento Escala de Expectativas de Futuro en la Adolescencia (EEFA). Se analizó una muestra compuesta por 1125 participantes de ambos sexos procedentes de la provincia de Cádiz, con edades comprendidas entre los 11 y 15 años. A partir del Análisis Factorial Exploratorio (AFE), realizado con la submuestra $1(n=551)$, se identificaron cuatro factores: Expectativas económi co/laborales, Expectativas académicas, Expectativas de bienestar personal y Expectativas familiares. Mediante un Análisis Factorial Confirmatorio (AFC), usando la submuestra $2(n=574)$, se confirmó la estructura factorial de la escala, cuyo modelo presentaba un buen ajuste. Los análisis de los ítems y de fiabilidad demostraron una aceptable consistencia interna de la escala. Para la obtención de otras evidencias de validez, los datos obtenidos mediante el instrumento EEFA se contrastaron con otros instrumentos que evalúan autoestima y satisfacción vital, esperando obtener relaciones positivas entre los mismos. Se concluye que el instrumento EEFA, compuesto por 14 ítems, posee propiedades psicométricas adecuadas, considerándose un instrumento válido para evaluar las expectativas de futuro que presentan los adolescentes.

Palabras clave: adolescencia; expectativas de futuro; propiedades psicométricas; autoestima; satisfacción vital.
\end{abstract}

\section{Introducción}

El concepto de expectativas de futuro hace referencia a la medida en que la persona espera que ocurra un evento, influyendo en la planificación y establecimiento de objetivos, guiando así la conducta y el desarrollo (Bandura, 2001; Nurmi, 1991; Seginer, 2008). Aunque la capacidad de orientarnos hacia el futuro se observa desde la infancia, su forma, contenido y función cambiarán con la edad, y su capacidad para autodirigirnos adquirirá mayor importancia en la adolescencia (Lewin, 1939). La preparación para la vida adulta es en sí una tarea evolutiva adolescente que explica la especial relevancia de la orientación futura (Dreher y Oerter, 1986). Se trata de un período especialmente importante en la construcción de un proyecto vital, significativo y diferenciado, siendo ésta una de las tareas del desarrollo a realizar (Díaz Morales y Sánchez López, 2002). Es a partir de los 12 años, aproximadamente, cuando la persona comienza a adquirir un claro sentido del futuro (Oppenheimer, 1987), crece su preocupación por la toma de decisiones sobre su futuro (Jiménez, 2011), y comienza a tomarlas (Dryfoos, 1990). Las expectativas futuras son una base para el establecimiento de metas, exploración, planificación y toma de decisiones (Seginer, 2000). Son esenciales para prosperar a través de la adolescencia y se conciben como una vía positiva hacia la edad adulta (Snyder, 2002). En definitiva, durante esta etapa se

* Dirección para correspondencia [Correspondence address] Yolanda Sánchez-Sandoval*. Departamento de Psicología. Facultad de Ciencias de la Educación. Av. República Saharaui s/n. 11519 Puerto Real, Cádiz (España). E-mail: yolanda.sanchez@uca.es
Title: Development and validation of the Adolescent Future Expectations Scale.

Abstract: The aim of this study is to design and validate the Adolescent Future Expectations Scale. We analyze a sample consisting of 1125 participants of both sexes, from Cádiz, with an age range between 11 and 15 years old. Exploratory factorial analysis was performed with the sample $1 \quad(n=551)$ and it resulted in four factors: economic expectations, academic expectations, expectations of personal well-being and family expectations. The application of the confirmatory factor analysis was performed with the sample $2(n=574)$. The factor structure of the scale was confirmed. The model showed a good fit. The reliability and item analyses showed that the scale had good internal consistency. With the aim of determining construct validity, the data obtained by the Adolescent Future Expectations Scale were compared with other instruments that assess self-esteem and life satisfaction. We expected to get positive relationship between them. It was concluded that the 14 -item scale is a valid instrument to evaluate adolescents' future expectations.

Key words: adolescence; future expectations; psychometric properties; self-esteem; life satisfaction. atribuye una gran importancia al futuro, con respecto al cumplimiento de las aspiraciones y proyectos (Laghi, Pallini, D'Alessio, \& Baiocco, 2011). Como se ha indicado, la perspectiva de tiempo puede influir en las acciones y decisiones de los individuos y, como tales, puede ser un requisito previo para empezar a construir la identidad de uno, sobre todo durante la adolescencia (Laghi, Baiocco, Lonigro, Capacchione, y Baumgartner, 2012).

Los intereses de los adolescentes, con respecto a su futuro, se relacionan con varias dimensiones. A pesar de diferentes condiciones físicas, culturales, y creencias, los adolescentes crecen en entornos con cuatro dominios comunes sobre la vida futura: educación, trabajo, matrimonio/familia y autopreocupaciones (Bandura, 2006; Díaz Morales y Sánchez López, 2002; Nurmi, 1991). Otros estudios (Nuttin y Lens, 1985) indican que existen categorías principales en el nivel de representación mental del sujeto, como: el Self (aspectos individuales), las otras personas (reciprocidad, relación), objetos económicos y realidades conceptuales (constructos y valores). En un estudio longitudinal con adolescentes de 11 a 15 años (Nurmi, 1989), se utilizó un cuestionario de deseos y temores sobre el futuro, donde se encontró que éstos se interesaban principalmente en temas educacionales, familia y posesiones materiales. Estos resultados coinciden con los de otros autores (Díaz Morales y Sánchez López, 2002).

Teniendo en cuenta la anterior revisión, consideramos fundamental investigar las expectativas de futuro de los adolescentes en función a las dimensiones encontradas en la literatura. Por tanto, se ha efectuado una revisión de aquellos instrumentos ya existentes con respecto a dicho tema. Sin embargo, como consecuencia de las distintas aproximaciones 
teóricas al concepto de expectativas y orientación futura, y de las diferentes definiciones conceptuales y operacionales, encontramos muy diversa metodología para evaluarla (Del Río-González y Nidia Herrera, 2006). Entre ellas no obtuvimos ninguna acorde a nuestro objetivo, como es una escala breve y de fácil comprensión para los participantes de este estudio. Así, para evaluar la perspectiva de tiempo, se han utilizado cuestionarios estructurados y tests (Fingerman y Perlmutter, 1995; Strathman, Gleicher, Boninger y Edwards, 1994; Zimbardo y Boyd, 1999), además de pruebas de tipo proyectivo y métodos más cualitativos, como entrevistas (McCabe y Barnett, 2000). De todos estos instrumentos, uno de los más utilizados es el Inventario de Perspectiva de Tiempo de Zimbardo (Jackson, Fritch, Nagasaka y Pope, 2003; Honora y Freeman, 2001; Petry, Bickel y Arnett, 1998; Rothspan y Read, 1996; Wills, Sandy y Yaeger, 2001; Zimbardo y Boyd, 1999). Este instrumento es uno de los más rigurosamente diseñados y evaluados (Zimbardo y Boyd, 1999). Sin embargo, no es específico para adolescentes y, hasta donde se reporta en la literatura revisada, no ha sido adaptado al español. Aquel que más se acerca a nuestro objetivo es el Cuestionario de Metas para Adolescentes (Sanz de Acedo Lizarraga, Ugarte y Lumbreras Bea, 2003), pero se trata de una escala demasiado extensa. Otro de los instrumentos más utilizado es la Escala de Actitudes Temporales (TAS) de Nuttin y Lens (1985), basado en la técnica de Diferencial Semántico. Destacamos también el Hopeful Future Expectations (Lerner et al, 2011), validado con una muestra de adolescentes estadounidenses, y que tomamos como referencia para elaborar el nuestro propio.

Una vez comprobada la carencia e inadecuación de los instrumentos para nuestra investigación, nos embarcamos en el propósito de construir una escala que tuviera como constructo objeto de estudio las expectativas de futuro de los adolescentes españoles. La importancia de nuestro instrumento radica en su brevedad y en su lenguaje adaptado a los adolescentes. El objetivo de este trabajo es presentar el proceso de creación y validación de la Escala de Expectativas de Futuro en la Adolescencia (EEFA).

Además del instrumento diseñado en este trabajo, con el fin de estudiar las evidencias de validez, consideramos oportuno medir otros conceptos como autoestima y satisfacción vital. La principal razón es la asociación existente entre tales variables, defendidas por numerosos estudios. Algunos de éstos indican que ciertos constructos del self son importantes en la tendencia de los adolescentes a orientarse a sí mismos hacia el futuro, como autoevaluación, propia estabilidad y autoestima (Seginer, 2009). De igual modo, se ha corroborado la relación entre actitud temporal de los adolescentes y variables de personalidad como optimismo (Nurmi, 2004), desempeño, autocontrol, ansiedad o autoestima (Lennings, Burns y Cooney, 1998). Por otro lado, se ha encontrado una asociación importante entre una larga Perspectiva de Tiempo Futuro, una actitud positiva hacia el futuro y altos niveles de satisfacción con la vida (Thomae, 1981). Asimismo, se ha explorado la relación entre la satisfacción en diferentes áreas vi- tales y la valoración sobre el grado de cumplimiento de los objetivos de vida en el presente y el futuro (Castro y Sánchez López, 2000). Los resultados muestran asociaciones entre la satisfacción y el logro de objetivos vitales.

A continuación se especifican las fases de construcción y validación de la escala.

\section{Método}

\section{Participantes}

La muestra estuvo compuesta por 1125 participantes que cursaban $1^{\circ}$ de E.S.O., 574 eran niños (51\%) y 551 niñas (49\%), con edades comprendidas entre 11 y 15 años $M=$ 12.46, DT =0.69). Como se indicará más adelante, la muestra se dividió aleatoriamente en dos submuestras, para realizar respectivamente un AFE y un AFC. La submuestra 1, estaba formada por 551 personas, de las que $55.2 \%$ son chicos y el $44.8 \%$ chicas. La media de edad de este grupo es de 12.46 años $(D T=0.68)$. En la submuestra 2 participaron 574 personas ( $47 \%$ chicos y $53 \%$ chicas) con una media de edad de 12.46 años $(D T=0.71)$. Participaron los sujetos que no tenían ningún dato perdido entre las medidas cumplimentadas.

La selección de la muestra se hizo teniendo en cuenta los siguientes criterios: a) titularidad del centro (público / privado-concertado), b) tamaño de la población (grande, más de 90000 habitantes / mediana, menos de 90000 habitantes), y c) nivel socioeconómico de las familias (medio/medio-bajo). Finalmente, los participantes proceden de 48 aulas del primer curso de Educación Secundaria Obligatoria de once centros educativos de la provincia de Cádiz, de los cuales el 45.45\% eran centros privados concertados y el $54.54 \%$ públicos.

La recogida de datos se realizó en los cursos académicos 2011/2012 y 2012/2013, durante los meses de mayo, junio, noviembre y diciembre de 2012 y mayo y junio de 2013. La participación de los adolescentes fue totalmente voluntaria, además se mantuvo el anonimato de las respuestas dadas.

\section{Instrumentos}

La Escala de Expectativas de Futuro en la Adolescencia (EEFA)

Elaboración de la escala. Teniendo en cuenta la revisión de la literatura, y tras el análisis de otras medidas, se realizó una versión preliminar del instrumento que incluyera las dimensiones del concepto Expectativas de Futuro consideradas más relevantes y necesarias. Nuestra decisión fue que el instrumento a elaborar debía contener la evaluación de las expectativas de los chicos y chicas con respecto, al menos, a los siguientes ámbitos: material, académico, laboral, familiar, social, y de la salud y seguridad.

Para la elaboración de los ítems se tuvieron en cuenta, además de la revisión de la literatura pertinente, las definiciones del constructo y algunos ítems presentes en otras es- 
calas existentes (como la de Lerner et al, 2011), además de otros de elaboración propia.

La escala fue revisada y comentada con un grupo de expertos, confirmando así la versión preliminar de la misma, compuesta por 20 ítems que respondían a las dimensiones señaladas anteriormente. Tras el análisis métrico de los ítems, fue recomendable eliminar 6 de ellos (bajas correlaciones, aumentos del alfa de Cronbach si se elimina ítem,...). La versión definitiva de la escala quedó compuesta, por tanto, por 14 ítems.

Versión definitiva. Esta escala evalúa las expectativas de futuro que tienen los adolescentes, y permite conocer cómo creen que será su futuro imaginándose a sí mismos dentro de unos años. Se trata de un autoinforme compuesto por diferentes ítems, a responder con una escala Likert de cinco puntos, que forman parte de una serie de dimensiones fundamentales en las expectativas de los adolescentes. La puntuación de cada ítem indica el grado de probabilidad de que ocurran o no ciertas cosas en su futuro, con el siguiente formato de respuesta: 1 = "estoy seguro/a de que no ocurrirán", $2=$ "es difícil que ocurra", 3 = "puede que sí/puede que no", $4=$ "probablemente ocurra" y $5=$ "estoy seguro/a de que ocurrirá". En la Tabla 1 se presenta la escala definitiva.

Tabla 1. Versión definitiva de la Escala de Expectativas de Futuro en la Adolescencia

Nos gustaría conocer cómo crees que será tu futuro. Imagínate a ti mismo dentro de algún tiempo, por ejemplo cuando pasen algunos años, $y$ contesta a las siguientes cuestiones

¿Cómo de probable crees que ocurrirán las siguientes cosas?

Estoy seguro/a de que no ocurrirá (1)

Es difícil que ocurra (2)

Puede que sí/puede que no (3)

Probablemente ocurra (4)

Estoy seguro/a de que ocurrirá (5)

1. Terminaré los estudios de Secundaria

2. Encontraré trabajo

3. Tendré un coche

4. Terminaré Bachillerato o un Ciclo Formativo

5. Encontraré un trabajo que me guste

6. Tendré una vida familiar feliz

7. Seré respetado por los otros

8. Me sentiré seguro

9. Tendré una casa

10. Realizaré estudios universitarios

11. Encontraré un trabajo bien remunerado

12. Encontraré una persona con quien formar una pareja feliz y estable

13. Seré feliz

14. Tendré hijos

A partir de las definiciones del constructo y, tras la revisión de la literatura y otras escalas existentes, los ítems se agruparon en los siguientes factores:

1) Expectativas económico/laborales (Factor I). Este factor se compone de cinco ítems $(2,3,5,9,11)$ referente a las perspectivas laborales y a la adquisición de recursos para la cobertura de necesidades básicas y otras pertenencias.

2) Expectativas académicas (Factor II). Este factor, compuesto por tres ítems $(1,4,10)$, hace referencia al nivel de estudios que se espera alcanzar.

3) Expectativas de bienestar personal (Factor III). Este factor se compone de tres ítems $(7,8,13)$ que incluyen la posibilidad de desarrollar relaciones sociales y los aspectos que guardan relación con la salud y seguridad.

4) Expectativas familiares (Factor IV). Este factor, compuesto por tres ítems $(6,12,14)$, incluye la posibilidad de formar una familia estable, tener hijos, etc.

Para el estudio de las evidencias de validez se utilizaron los siguientes instrumentos:

Self Esteem Scale, RSE (Rosenberg, 1965), es una escala ampliamente utilizada para la medición global de este constructo, y desarrollada originalmente por este autor para la evaluación de la autoestima en adolescentes. Incluye 10 ítems cuyos contenidos se centran en los sentimientos de respeto y aceptación de sí mismo/a. Se trata de una escala tipo Likert, donde los ítems se corresponden a una escala de cuatro puntos $(1=$ "totalmente en desacuerdo", $2=$ "en desacuerdo", $3=$ "de acuerdo", 4= "totalmente de acuerdo"). La puntuación general de autoestima se obtiene a partir del sumatorio de todos los ítems. En nuestro estudio, la escala muestra una aceptable consistencia interna, con un coeficiente alfa de Cronbach de .70 .

Escala de Satisfacción con la vida, SLSS (Pavot y Diener, 1993). Se trata de una escala destinada a evaluar la satisfacción global con la vida; concretamente mide el componente cognitivo del bienestar subjetivo. Consiste en un conjunto de 7 afirmaciones respecto a las cuales los participantes deben indicar el grado en que están o no de acuerdo con las mismas, presentándose para ello una escala de 6 opciones: "Totalmente en desacuerdo", "bastante en desacuerdo", "algo en desacuerdo", "algo de acuerdo", "bastante de acuerdo" y "totalmente de acuerdo". En general, se trata de un instrumento breve y preciso para la medición de la satisfacción vital global de los adolescentes. En este estudio, esta escala muestra una alta consistencia interna, con un coeficiente alfa de Cronbach de .81. La fiabilidad y validez de estas dos escalas han sido demostradas en otros trabajos con población adolescente española (e.g. Buelga, Cava y Musitu, 2012).

\section{Procedimiento}

Como paso previo, se confirmó la colaboración y aceptación de los diferentes centros para participar en la investigación. A través de los centros, se envió una carta a los padres informándoles de la investigación y solicitándoles el consentimiento informado por escrito para la participación de sus hijos. Tras la depuración inicial de la escala, la versión de 14 ítems seleccionados para el estudio final fue administrada en horario escolar por personal entrenado para tal fin, cuya duración fue de aproximadamente 10 minutos. En las instruc- 
ciones a los participantes se indicó que la participación era voluntaria, que el cuestionario debía cumplimentarse de manera individual y en el horario facilitado, y que en todo momento se mantendría la confidencialidad y el anonimato de los datos, asignando para ello un código sólo conocido por los alumnos.

\section{Análisis de datos}

Se empleó el software PASW Statistics-18 para obtener las propiedades psicométricas de los ítems y realizar el Análisis Factorial Exploratorio (AFE). Mediante un Análisis Factorial Confirmatorio (AFC), con el uso del software $\mathrm{R}$, se confirmó la estructura factorial de la escala. Para ello, la muestra se dividió aleatoriamente en dos mitades a través del programa SPSS, independientemente de la edad y el sexo de los participantes. Los casos con datos ausentes fueron omitidos.

Con la submuestra 1 se realizó el estudio métrico de los ítems y un AFE, para testar la estructura teórica de la escala, usando el método de ejes principales y rotación quartimax. Este método incorpora a la explicación factorial tanto la varianza común como la específica. Aunque al trabajar con escalas ordinales se recomienda emplear modelos de análisis basados en correlaciones policóricas (Brown, 2006), se ha utilizado un AFE ya que, salvo en casos extremos (distribuciones marcadamente asimétricas o asimetrías en ambas direcciones), las aproximaciones suelen ser aceptables cuando se usan escalas de respuesta graduada con 5 ó más categorías (Ferrando y Anguiano-Carrasco, 2010), como es nuestro caso. La comunidad científica utiliza este método actualmente para el análisis de escalas de tipo Likert que evalúan variables de personalidad en la infancia y adolescencia (BernabéValero, García-Alandete, Gallego-Pérez, 2014; Espada et al., 2013; García-Olcina, Piqueras y Martínez-González, 2014; Labrador, Villadangos, Crespo y Becoña, 2013; Ochoa-Meza, Sierra, Pérez-Rodrigo y Aranceta-Bartrina, 2014; Oros, 2014; Ruiz-Juan, 2014).

Con la submuestra 2 se validó la estructura factorial obtenida anteriormente, mediante un AFC. El método de estimación fue máxima verosimilitud. Para determinar el ajuste del modelo propuesto, se presenta una combinación de aquellos índices más utilizados en la investigación. En primer lugar, se tuvo en cuenta la prueba de bondad de ajuste $\chi 2$. Al tratarse de un parámetro sensible a $n$, se han hallado otros indicadores para evaluar el ajuste global. Éstos han sido el indice de ajuste comparado (CFI) y el indice de Tucker-Lewis (TLI), cuyos valores iguales o superiores a .90 se interpretarán como propios de un ajuste adecuado. También se ha interpretado el residuo cuadrático medio estandarizado (SRMR), considerando un ajuste adecuado si su valor es inferior a .05 y el error cuadrático medio de aproximación (RMSEA), considerando indicador de un ajuste adecuado todo valor igual o inferior a .08.

Posteriormente, se procedió a la construcción de las subescalas, mediante las puntuaciones medias de los ítems que las componen. Se realizaron los análisis de correlación de Pearson entre los ítems y cada una de las subescalas del instrumento. Además, se llevaron a cabo los análisis de fiabilidad y consistencia interna de la escala y subescalas (correlación ítem-total corregida), mediante el coeficiente alfa de Cronbach.

Por último, se han realizado correlaciones bivariadas con otras variables que proporcionen evidencias externas de la de validez. Se utilizó el coeficiente de correlación de Pearson entre las puntuaciones totales de la Escala de Expectativas de Futuro en la Adolescencia, escala de Autoestima y escala de Satisfacción vital.

\section{Resultados}

\section{Análisis métrico de ítems}

Realizado el análisis psicométrico de los 20 ítems planteados en la versión preliminar, y dada la ausencia de un resultado satisfactorio (baja fiabilidad, menor coeficiente de correlación, problemas en la redacción y comprensión de los ítems), se procedió a eliminar 6 de ellos.

En la Tabla 2 se muestran propiedades psicométricas de cada uno de los 14 ítems que componen la versión final de la $E E F A$, tales como la media, la desviación típica, índice de discriminación (correlación ítem total corregida) y alfa de Cronbach si el ítem es eliminado de la escala. Como puede comprobarse, la eliminación de cualquiera de los ítems haría disminuir la fiabilidad total de la escala (.85). Asimismo, los datos muestran altos coeficientes de correlación de cada ítem con la dimensión teórica a la que pertenece.

\section{Evidencias de validez basadas en la estructura in- terna: AFE y AFC}

\section{Análisis Factorial Exploratorio}

Se realizó un Análisis Factorial Común (AFE) con el método de ejes principales (rotación Quartimax), a partir de la submuestra 1. Previamente a la interpretación del análisis factorial, se aplicaron las pruebas KMO y la prueba de esfericidad de Barlett.

La solución factorial obtenida mediante el criterio de retención de factores de Kaiser (1974) estuvo formada, en principio, por tres factores que explicaban el $45.64 \%$ de la varianza. Mostró valores adecuados de $\mathrm{KMO}(\mathrm{KMO}=.86)$ y esfericidad también estadísticamente significativa $\left(\chi^{2}(91)=\right.$ $2547.399, p<.000)$. Ya que la regla de Kaiser puede llegar a sobredimensionar (o infradimensionar) la estructura factorial resultante, hemos completado este análisis con el estudio del gráfico de sedimentación. Finalmente, se obligó a una solución de cuatro factores ya que el gráfico de sedimentación así lo aconsejaba (Figura 1) y, además, son cuatro las dimensiones teóricas del constructo analizado que se tuvieron en cuenta para la redacción de los ítems, a partir de la literatura existente. 


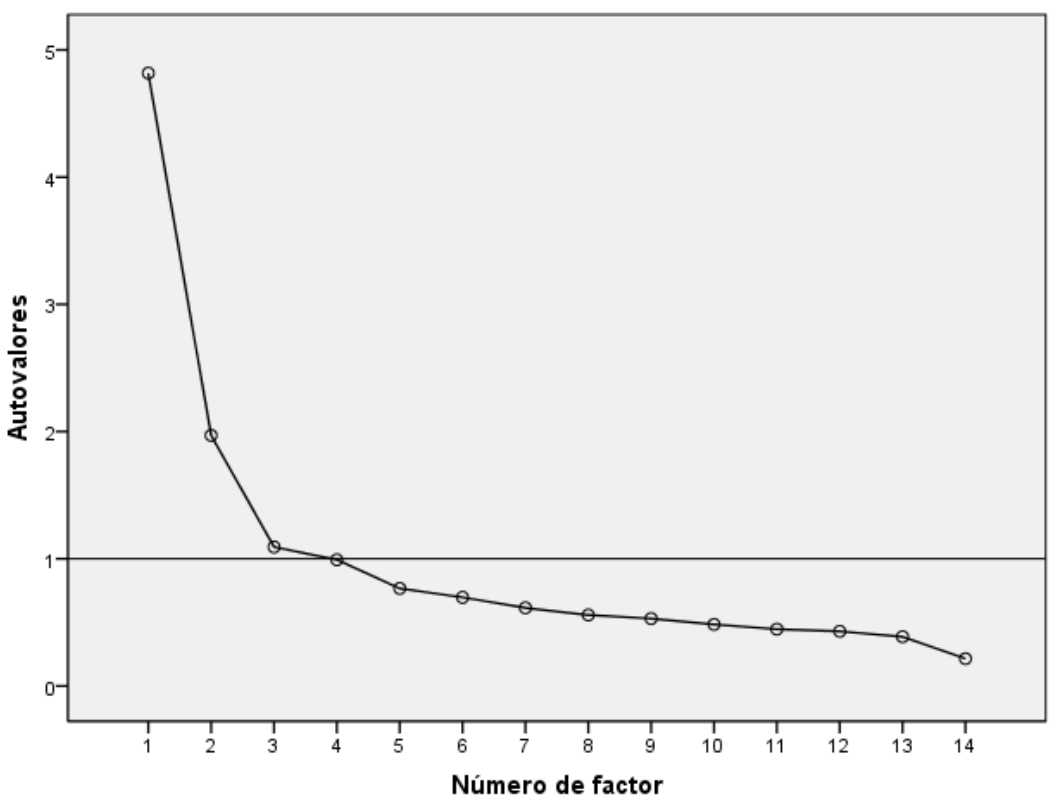

Figura 1. Gráfico de Sedimentación.

En nuestro modelo podemos comprobar que a partir del factor número cuatro el autovalor comienza a ser inferior a la unidad. El porcentaje de la varianza explicada, acumulada por estos primeros cuatro factores, es de $49.72 \%$. Podemos considerar, por tanto, que éste puede ser un valor suficientemente alto para estimar que cuatro es un número de factores adecuado.

En la determinación de los elementos de cada factor se consideró la regla habitual de saturaciones mínimas superior a .30, criterio para considerar que un elemento es indicador de factor. Como puede apreciarse en la Tabla 3, aparece una solución de los factores que se mantiene estable (se han su- primido las cargas factoriales menores a .30). Los cuatro factores tienen una interpretación teórica coherente en función de la dimensión que pretenden medir. El primer factor explica el $25.39 \%$ y, por el significado de los ítems con mayor peso en el mismo, hemos denominado Expectativas económico/laborales. El segundo factor, Expectativas académicas, explica el $13.17 \%$ de la varianza. El tercer factor, Expectativas de bienestar personal, explica el $5.60 \%$ de la varianza. Por último, el cuarto factor, Expectativas familiares, explica el 5.54\% de la varianza. Puntuar alto en cada uno de estos factores indicaría tener mayores expectativas en el ámbito al que se refiera.

Tabla 2. Estadísticos descriptivos, fiabilidad e índices para cada uno de los ítems de la escala.

\begin{tabular}{|c|c|c|c|c|c|}
\hline Ítems & Media & $\begin{array}{l}\text { Desviación } \\
\text { Típica }\end{array}$ & $\begin{array}{l}\text { Correlación elemento-total I } \\
\text { corregida }\end{array}$ & $\begin{array}{l}\text { Fiabilidad eliminando } \\
\text { el ítem } \\
\end{array}$ & $\begin{array}{c}\text { Correlación ítem- } \\
\text { subescala }\end{array}$ \\
\hline 1. Terminaré los estudios de Secundaria & 4.53 & .90 & .45 & .835 & .79 \\
\hline 2. Encontraré trabajo & 4.17 & .78 & .55 & .830 & .76 \\
\hline 3. Tendré un coche & 4.23 & .83 & .47 & .834 & .69 \\
\hline 4. Terminaré bachillerato o Ciclo Formativo & 4.04 & 1.12 & .50 & .833 & .91 \\
\hline 5. Encontraré un trabajo que me guste & 4.10 & .93 & .61 & .825 & .77 \\
\hline 6. Tendré una vida familiar feliz & 4.50 & .73 & .46 & .835 & .71 \\
\hline 7. Seré respetado por los otros & 4.14 & .83 & .44 & .836 & .79 \\
\hline 8. Me sentiré seguro & 4.40 & .73 & .46 & .835 & .79 \\
\hline 9. Tendré una casa & 4.50 & .73 & .55 & .830 & .69 \\
\hline 10. Realizaré estudios universitarios & 3.88 & 1.23 & .50 & .835 & .90 \\
\hline 11. Encontraré un trabajo bien remunerado & 3.96 & .87 & .60 & .826 & .77 \\
\hline $\begin{array}{l}\text { 12. Encontraré una persona con quien formar } \\
\text { una pareja feliz y estable }\end{array}$ & 4.38 & .79 & .44 & .836 & .81 \\
\hline 13. Seré feliz & 4.63 & .60 & .45 & .837 & .69 \\
\hline 14. Tendré hijos & 4.09 & .96 & .33 & .844 & .80 \\
\hline
\end{tabular}


Tabla 3. Matriz de configuración.

\begin{tabular}{|c|c|c|c|c|}
\hline Ítems & F1 & F2 & F3 & $\mathrm{F} 4$ \\
\hline 05 Encontraré un trabajo que me guste & .70 & & & \\
\hline 11 Encontraré un trabajo bien remunerado & .69 & & & \\
\hline 02 Encontraré trabajo & .69 & & & \\
\hline 09 Tendré una casa & 60 & & & \\
\hline 03 Tendré un coche & .58 & & & \\
\hline 04 Terminaré Bachillerato o un Ciclo Formativo & & .89 & & \\
\hline 10 Realizaré estudios universitarios & & .76 & & \\
\hline 01 Terminaré los estudios de Secundaria & & .61 & & \\
\hline 07 Seré respetado por los otros & & & .40 & \\
\hline 08 Me sentiré seguro & & & .49 & \\
\hline 13 Seré feliz & & & .41 & \\
\hline 14 Tendré hijos & & & & .57 \\
\hline 12 Encontraré una persona con quien formar una pareja feliz y estable & & & & .56 \\
\hline 06 Tendré una vida familiar feliz & & & & .39 \\
\hline Valor propio & 4.81 & 1.97 & 1.09 & .99 \\
\hline Porcentaje de varianza explicada & 25.39 & 13.17 & 5.60 & 5.54 \\
\hline
\end{tabular}

Nota: Método de extracción: Análisis de Ejes Principales. Método de Rotación: Quartimax.

F1: Expectativas económico/laborales; F2: Expectativas académicas; F3: Expectativas de bienestar personal; F4: Expectativas familiares.

\section{Análisis Factorial Confirmatorio}

Tras el AFE, los datos de la segunda mitad de la muestra fueron sometidos a un AFC para evaluar el ajuste del modelo, cumpliendo con los requisitos de ajuste exigibles.

Los resultados del análisis confirmatorio respecto al modelo esperado, pusieron de manifiesto el buen ajuste del modelo propuesto. Los índices de ajuste obtenidos son: $\chi^{2}$
$=159$, g.l. $=71, \mathrm{p}=.000 ;$ RMSEA $=.047$ (intervalo de confianza del 90\%:.037-.057); SRMR=.040; CFI= .96; TLI $=.95$. La Figura 2 muestra los coeficientes factoriales estandarizados del modelo. Como resultados generales del AFC, se muestra una bondad de ajuste aceptable con respecto al modelo teórico de acuerdo con el diagrama de ruta de la Figura 2.

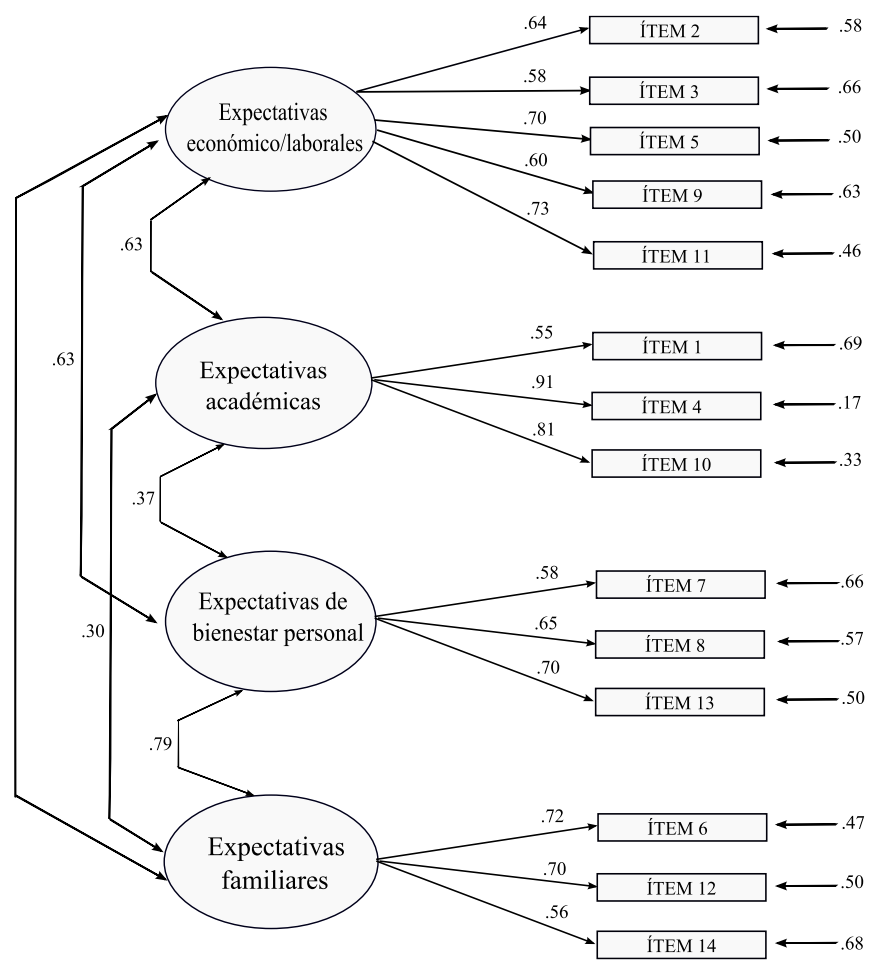

Figura 2. Modelo dimensional de la EEFA

Nota. Las correlaciones entre cada dimensión vienen representadas con flechas bidireccionales. Los coeficientes $B$ estandarizados se representan encima de cada una de las flechas unidireccionales. La variabilidad explicada de los ítems viene indicada al lado de las flechas unidireccionales. 


\section{Estimación de la fiabilidad}

El coeficiente alfa de Cronbach fue de .85. En la Tabla 4 se presentan los resultados de dichos cálculos. Como se puede observar, en los cuatro factores se obtienen coeficientes de una aceptable consistencia interna, con respecto al número de ítems que contienen, mostrando resultados satisfactorios. Así, la consistencia interna de los factores fue de 79 (Factor I), .81 (Factor II), .65 (Factor III) y .68 (Factor IV).

Tabla 4. Análisis de consistencia interna.

\begin{tabular}{lccc}
\hline & Ítems & $\begin{array}{c}\text { Correlación } \\
\text { elemento-total corregida }\end{array}$ & $\begin{array}{c}\text { Alfa si se eli- } \\
\text { mina el ítem }\end{array}$ \\
\hline F1: Expectativas eco- & 02 & .588 & .742 \\
nómico/laborales & 05 & .500 & .770 \\
$\alpha=.79$ & 09 & .599 & .738 \\
& 11 & .536 & .759 \\
F2: Expectativas aca- & 01 & .617 & .732 \\
démicas & 04 & .560 & .857 \\
$\alpha=.81$ & 10 & .771 & .641 \\
F3: Expectativas de & 07 & .719 & .704 \\
bienestar personal & 08 & .443 & .613 \\
$\alpha=.65$ & 13 & .519 & .494 \\
F4: Expectativas fami- & 06 & .464 & .579 \\
liares & 12 & .576 & .638 \\
$\alpha=.68$ & 14 & .480 & .497 \\
Total de EEFA & & & .637 \\
$\alpha=.85$ & & & \\
\hline
\end{tabular}

\section{Estudio de evidencias basadas en la relación con} otras variables

Las fuentes para conseguir evidencias sobre la validación de constructo de la EEFA se han centrado en su conceptualización teórica, de la que se derivan las diferentes relaciones que mantiene esta variable objeto de estudio con medidas de otras variables importantes en este tema en cuestión, tales como autoestima y satisfacción vital del propio sujeto.

De acuerdo con los planteamientos iniciales, los resultados obtenidos de los análisis de correlaciones realizados entre las puntuaciones totales del instrumento EEFA y Escala de Autoestima, muestran que existe una correlación positiva significativa $(r=.48 ; p<.001)$ entre los mismos. Así, cuanto mayores son las expectativas de futuro de los adolescentes, mayor es también su autoestima. Igualmente, y como se esperaba teóricamente, encontramos correlaciones positivas y estadísticamente significativas entre las puntuaciones en el instrumento EEFA y las obtenidas en la escala de Satisfacción con la vida $(r=.45 ; p<.001)$. Por tanto, cuanto mayores expectativas de futuro, mayor satisfacción con la vida manifiestan los adolescentes.

Se han hallado correlaciones positivas significativas entre las cuatro subescalas del instrumento EEFA y el total de la Escala de Autoestima (expectativas económico/laborales $(r=.37$; $p<.001)$, expectativas académicas $(r=.31 ; p<.001)$, expectativas de bienestar personal $(r=.48 ; p<.001)$ y expectativas familiares $(r=.29$; $p<.001)$, respectivamente).

De igual modo, se han obtenido correlaciones positivas significativas entre dichas subescalas y el total de la Escala de Satisfacción con la vida (expectativas económico/laborales $(r=.33$; $p<.001)$, expectativas académicas $(r=.30 ; p<.001)$, expectativas de bienestar personal $(r=.43 ; p<.001)$ y expectativas familiares $(r=.29$; $p<.001)$, respectivamente).

\section{Discusión y conclusiones}

El objetivo de este trabajo ha sido la elaboración y validación de una escala para medir las expectativas de futuro de los adolescentes, con el fin de suplir un vacío en cuanto a instrumentos de evaluación para este período del ciclo vital. El instrumento resultante se denomina Escala de Expectativas de Futuro en la Adolescencia (EEFA) y se validó en una muestra de 1125 participantes de la provincia de Cádiz. Se analizaron las propiedades psicométricas del instrumento, estudiando su estructura factorial (validez de constructo), consistencia interna y evidencias de validez. Para esto último se tuvieron en cuenta medidas de Autoestima y Satisfacción Vital, por ser variables teóricamente implicadas.

El estudio demostró que el cuestionario posee buenas propiedades psicométricas y se constituye en una alternativa válida y consistente para evaluar las expectativas de futuro de los adolescentes. Consideramos que la aportación de este instrumento da respuesta a una necesidad detectada en la investigación psicológica con respecto a la medida de la variable Expectativas de Futuro. Esta necesidad estaba generada por la falta de coordinación existente entre la definición teórica y operacional del constructo, así como por la multiplicidad de definiciones y métodos para medirlo.

En el presente estudio, el plan de análisis permitió abordar la estructura factorial de la escala. Se encontró que el instrumento EEFA posee una adecuada validez, resultando apropiada para ser aplicada a una muestra poblacional de adolescentes entre los 11 y 15 años, ya que su estructura factorial es consistente. Los análisis factoriales muestran una estructura compuesta por cuatro factores, con una alta coherencia desde el punto de vista teórico.

En cuanto a los resultados de consistencia interna, el instrumento presenta en cada una de las subescalas aceptables índices de fiabilidad, demostrando ser una herramienta fiable para evaluar las expectativas de futuro de los adolescentes, tanto a nivel global como considerando sus cuatro subescalas.

La escala está compuesta, finalmente, por cuatro factores que explican conjuntamente un $49.72 \%$ de la varianza. El primer factor, Expectativas económico laborales, así denominado por el significado de los ítems con mayor peso, explica el $25.39 \%$. Puntuar alto en esta dimensión, significa tener mayores expectativas con respecto al ámbito laboral (ocupación y profesión), posesiones materiales y objetos necesarios para cubrir las necesidades básicas. Se trata de una dimensión importante y de gran preocupación para los adolescentes en la 
actualidad. Hoy en día el joven ve con cierto pesimismo su incorporación al trabajo y su anhelada emancipación familiar, económica y social (Sanz De Acedo Lizarraga, Ugarte y Lumbreras Bea, 2003).

El segundo factor, Expectativas académicas (nivel de estudios que el sujeto espera alcanzar), explica el $13.17 \%$ de la varianza. Durante la etapa de Educación Secundaria Obligatoria, existe una creciente presión hacia el rendimiento que experimentan los adolescentes (Jiménez, 2011; Llamazares Sánchez, 2006), además de una preocupación en la toma de decisiones sobre su futuro que en breve deben tomar. Se trata de esfuerzos por adquirir una formación que les abra las puertas al mundo laboral, garantizando su desenvolvimiento personal y social futuro.

El tercer factor, Expectativas de bienestar personal, explica el $5.60 \%$ de la varianza. Los adolescentes que puntúan alto en esta dimensión esperan establecer buenas relaciones sociales y se imaginan como personas seguras y saludables. Algunos autores (Montoya y Landero, 2008) destacan que las interacciones sociales contribuyen a la satisfacción con la vida y al bienestar de los adolescentes, puesto que ofrecen al sujeto recursos y oportunidades para su realización personal.

Por último, el cuarto factor, Expectativas familiares, explica el $5.54 \%$ de la varianza. Los adolescentes que puntúan alto en este factor, poseen buenas expectativas referentes a la posibilidad de encontrar una pareja, formar una familia estable $\mathrm{y}$ tener hijos en el futuro.

Los análisis factoriales, con la submuestra 2, confirmaron dicha estructura factorial. Todas estas dimensiones coindicen, en gran medida, con lo que vienen defendiendo otros autores que muestran interés en esta línea de investigación. Como algunos indican, las esperanzas y expectativas de los adolescentes acerca de su futuro influyen en sus comportamientos presentes y se vinculan con las decisiones de su propia familia, educación y carrera (Nurmi, Poole y Kalakoski, 1994). Las dimensiones que se han encontrado en este estudio también se han encontrado, parcialmente, en las investigaciones llevadas a cabo por Gómez-Bustamante y Cogollo (2010). Éstos destacan la importancia de los cambios y transiciones producidos en la adolescencia, los cuales suponen modificaciones emocionales, familiares, académicos o laborales, que facilitan el logro de la identidad personal. En esta misma línea, Wade (2008) también destaca la importancia del

\section{Referencias}

Bandura, A. (2006). Toward a psychology of human agency. Perspectives on Psychological Science, 1(2), 164-180. doi: 10.1111/j.17456916.2006.00011.x

Bernabé-Valero, G., García-Alandete, J. y Gallego-Pérez, J. F. (2014). Construcción de un cuestionario para la evaluación de la gratitud: el Cuestionario de Gratitud-20 ítems (G-20). Anales de Psicología, 30(1), 278-286. doi: 10.6018/analesps.30.1.135511

Brown, T. A. (2006). Confirmatory factor analysis for applied research. New York: The Guilford Press. apoyo familiar y relaciones con los compañeros en la conformación de planes y expectativas de futuro de los adolescentes.

La EEFA muestra otras evidencias externas de validez, mostrando altas correlaciones entre las propias Expectativas de Futuro y variables como Autoestima y Satisfacción Vital. Estas asociaciones adquieren mayor fiabilidad al comprobar que en otros estudios se han obtenido resultados semejantes. Como indica García-Viniegras (2005), otros autores también señalan la satisfacción que se deriva de los logros alcanzados en la vida, acorde con los propósitos o proyectos personales, destacando la estrecha relación entre satisfacción y felicidad. Asimismo, otros estudios muestran que el logro de las metas que el individuo se plantea contribuye a la realización del plan de vida y, por tanto, a elevar su sensación de bienestar o satisfacción. En este sentido, la satisfacción de necesidades, deseos o la obtención de metas proporciona emociones altamente positivas (Díaz Morales y Sánchez López, 2002). Referente a la autoestima, como destacan algunos autores (García-Viniegras, 2005), una autoestima elevada es una valoración positiva de nosotros mismos, que produce satisfacción y disfrute personal, y a la vez puede producir conductas encaminadas a la obtención de metas (adecuado nivel de aspiración) que producen satisfacción y bienestar, lo cual refuerza la autoestima.

En conclusión, la EEFA, con tan solo 14 ítems, pudiéndose realizar en 10 minutos, es capaz de evaluar las expectativas de futuro de los adolescentes. No obstante, es evidente que el trabajo presenta también algunas limitaciones que no podemos dejar de señalar. Destacamos como limitación el uso exclusivo de autoinformes. En este sentido, las respuestas aportadas por los participantes podrían verse influenciadas por la deseabilidad social, por lo que podría afectar a las propiedades psicométricas de la escala así como a la interpretación de los resultados obtenidos. Para futuras investigaciones pretendemos utilizar este instrumento en muestras más amplias así como analizar su relación con otras variables psicosociales señaladas por la literatura. Por último, pretendemos hacer un acercamiento longitudinal, entrevistando a los mismos participantes, con el fin de evaluar la estabilidad y evolución del concepto. Sería interesante ampliar este trabajo con informes procedentes de fuentes externas al adolescente como podrían ser sus profesores y familiares.

Buelga, S., Cava, M. J. y Musitu, G. (2012). Reputación social, ajuste psicosocial y victimización entre adolescentes en el contexto escolar. Anales de Psicología, 28(1), 180-187.

Castro, A. y Sánchez-López, M. (2000). Objetivos de vida y satisfacción autopercibida en estudiantes universitarios. Psicothema, 12(1), 87-92.

Del Río-González, A. M. y Nidia Herrera, A. (2006). Desarrollo de un instrumento para evaluar perspectiva de tiempo futuro en adolescentes. Avances en Medición, 4, 47-60. 
Díaz Morales, J. y Sanchez López, M. (2002). Metas personales y satisfacción autopercibida. En G. Hernández (Ed.), Motivación animal y bumana (pp. 359-380). México: El Manual Moderno.

Dreher, E. y Oerter, R. (1986). Children's and adolescents' conceptions of adulthood: the changing view of a crucial developmental task. En R. K. Silbereisen, K. Eyferth y R. Rudinger (Eds.), Development as action in context: Problem behaviour and normal youth development (pp. 109-120). New York: Springer Verlag.

Dryfoos, J. G. (1990). Adolescents at Risk: Prevalence and Prevention. New York: Oxford University Press.

Espada, J. P., Ballester, R., Huedo-Medina, T. B., Secades-Villa, R., Orgilés, M. y Martínez-Lorca, M. (2013). Development of a new instrument to assess AIDS-related attitudes among Spanish Youngsters. Anales de Psicología, 29(1), 83-89. doi: 10.6018/analesps.29.1.132301

Ferrando, P. J. y Anguiano-Carrasco, C. (2010). El análisis factorial como técnica de investigación en psicología. Papeles del Psicólogo, 31(1), 18-33.

Fingerman, K. y Perlmutter, M. (1995). Future time perspective and life events across adulthood. The Journal of General Psychology, 122, 95-108.

García-Olcina, M., Piqueras, J. A. y Martínez-González, A. E. (2014). Datos preliminares de la validación del Cuestionario de Detección vía Web para los trastornos emocionales (DETECTA-WEB) en adolescentes españoles. Revista de Psicología Clínica con Niños y Adolescentes, 1(1), 69-77.

García-Viniegras, C. R. V. (2005). El bienestar psicológico: Dimensión subjetiva de la calidad de vida. Revista Electrónica de Psicología Iztacala, 8(2), 20.

Gómez-Bustamante, E. M. y Cogollo, Z. (2010). Factores predictores relacionados con el bienestar general en adolescentes estudiantes de Cartagena, Colombia. Revista de Salud Pública, 12(1), 61-70. doi: 10.1590/S0124-00642010000100006

Honora, D. y Freeman, J. (2001). Future time perspective as a correlate to academic achievement and school membership among urban African American adolescents. Adolescence, 42(167), 525538.

Jackson, T., Fritch, A., Nagasaka, T. y Pope, L. (2003). Procrastination and perceptions of past, present and future. Individual Differences Research, 1, 17-28.

Jiménez, T. I. (2011). Autoestima de riesgo y protección: Una mediación entre el clima familiar y el consumo de sustancias en adolescentes. Psychosocial Intervention, 20(1), 53-61. doi: $10.5093 /$ in $2011 \mathrm{v} 20 \mathrm{n} 1 \mathrm{a} 6$

Kaiser, H. F. (1974). An index of factorial simplicity. Psychometrika, 39, 31-36.

Labrador, F. J., Villadangos, S. M., Crespo, M. y Becoña, B. (2013). Desarrollo y validación del cuestionario de uso problemático de nuevas tecnologías (UPNT). Anales de Psicología, 29(3), 836-847. doi: 10.6018/analesps.29.3.159291

Laghi, F., Pallini, S., D'Alessio, M., y Baiocco, R. (2011). Development and validation of the efficacious self-presentation scale. Journal of Genetic Psychology, 172(2), 209-219. doi: 10.1080/00221325.2010.526975

Laghi, F., Baiocco, R., Lonigro, A., Capacchione, G., y Baumgartner, E. (2012). Family functioning and binge drinking among Italian adolescents. Journal of Health Psychology, 17(8), 1132-1141. doi: $10.1177 / 1359105311430005$

Lennings, C., Burns, A. y Cooney, G. (1998). Profiles of time perspective and personality: developmental considerations. Journal of Psychology, 132(6), 629-641.
Lerner, R. M., Lerner, J. V., Lewin-Bizan, S., Boyd, M. J., Kieluy Mueller, M., Schmid, K. L., Alberts Warren, A. y Bowers, E. (2011). The 4-H Study of positive youth development: past, present and future. Tufts University: Institute for Applied Research in Youth Development.

Lewin, K. (1939). Field theory and experiment in social psychology. American Journal of Sociology, 44, 868-896.

Llamazares Sánchez, L. (2006). Intereses académico profesionales y sociales de los jóvenes de entre 16 y 20 años de la ciudad de león. Humanismo y Trabajo Social, 5, 197-230.

McCabe, K. y Barnett, D. (2000). First comes work, then comes marriage: Future orientation among African American young adolescents. Family relations, 49, 63-70. doi: 10.1111/j.17413729.2000.00063.x

Montoya, B. y Landero, R. (2008). Satisfacción con la vida y autoestima en jóvenes de familias monoparentales y biparentales. Psicologia y Salud, 18(1), 117-122.

Nurmi, J. E. (1989). Development of orientation to the future during early adolescence: A four-year longitudinal study and two cross-sectional comparisons. International Journal of Psychology, 24(2), 195-214. doi: 10.1080/00207594.1989.10600042

Nurmi, J. E. (1991). How do adolescents see their future? A review of the development of future orientation and planning. Developmental Review, 11(1), 1-59. doi: 10.1016/0273-2297(91)90002-6

Nurmi, J. E. (2004). Socialization and self-development: channeling, selection, adjustment, and reflection. En R. M. Lerner y L. Steinberg (Eds.), Handbook of adolescent psychology, vol. 2 (pp. 85124). Hoboken, NJ: Wiley.

Nurmi, J. E., Poole, M. y Kalakoski, V. (1994). Age differences in adolescent future oriented goals, concerns, and related temporal extension in different sociocultural contexts. Journal of Youth and Adolescence, 23(4) 471-487.

Nutin, J. y Lens, W. (1985). Future time perspective and motivation: Theo$r y$ and research method. Location: Erlbaum.

Oppenheimer, L. (1987). Cognition and social variables in the plan of action. In S. H. Freidman, E. Kofsky-Scholnick y R. R. Cocking (Eds.) Blueprints for thinking: the role of planning in cognitive development. Cambridge: Cambridge University Press.

Pavot, W. y Diener, E. (1993). Review of the Satisfaction with Life Scale. Psychological Assessment, 5(2), 164-172. doi: 10.1007/97890-481-2354-4-5

Petry, N. M., Bickel, W. K. y Arnett, M. (1998). Shortened time horizons and insensitivity to future consequences in heroin addicts. Addiction, 93(5), 729-738. doi: 10.1046/j.13600443.1998.9357298.x

Rosenberg, M. (1965). Society and the adolescent selfimage. Princeton, NJ: Princeton University Press.

Rothspan, S. y Read, S. (1996). Present Versus Future Time Perspective and HIV Risk Among Heterosexual College Students. Health Psychology, 15(2), 131-134. doi: 10.1037/02786133.15.2.131

Sanz De Acedo Lizarraga, M. L., Ugarte, M. D. y Lumbreras Bea, M. V. (2003). Desarrollo y validación de un cuestionario de metas para adolescentes. Psicothema, 15(3), 493-499.

Seginer, R. (2000). Optimism correlates of adolescent future orientation: A domain-specific analysis. Journal of Adolescent Research, 15(3), 307-326. doi: 10.1177/0743558400153001

Seginer, R. (2009). Future Orientation. Developmental and Ecological Perspectives. Israel: Springer.

Snyder, C. R., Rand, K. L. y Sigmon, D. R. (2002). Hope theory: A member of the positive psychology family. En C. R. Snyder y S. 
J. Lopez (Eds.), Handbook of positive psychology (pp. 231-243). New York: Oxford University Press.

Strathman, A., Gleicher, F., Boninger, D. S. y Edwards, S. (1994). The consideration of future consequences: Weighing immediate and distant outcomes of behavior. The Journal of Personality and Social Psychology, 66(44), 742-752. doi: 10.1037/0022$\underline{3514.66 .4 .742}$

Thomae, H. (1981). Future Time Perspective and the Problem of Cognition/Motivation Interaction. En G. D'Ydewalle y W. Lens (Eds.), Cognition in Human Motivation and Learning (pp. 261274). Hillsdale, New Jersey: LEA.
Wade, J. (2008). The ties that bind: Support from birth families and substitute families for young people leaving care. British Journal of Social Work, 38, 39-54. doi: 10.1093/bjsw/bcl342

Wills, T. A., Sandy, J. M. y Yaeger, A. M. (2001). Time perspective and early-onset substance use: A model based on stress-coping theory. Psychology of addictive behaviors, 15(2), 118-125. doi: 10.1037/0893-164X.15.2.118

Zimbardo, P. G. y Boyd, J. N. (1999). Putting time in perspective: A valid, reliable individual-differences metric. Journal of Personality and Social Psychology, 77(6), 1271-1298. doi: 10.1037/0022$\underline{3514.77 .6 .1271}$

(Artículo recibido: 08-09-2014; revisado: 30-09-2014; aceptado: 10-10-2014) 